\title{
Automatic Boundary Delineation of Agricultural Fields in Multi temporal Satellite Imagery with Segmentation
}

\author{
R. Priya, Darsana K Gopidas
}

\begin{abstract}
A right difference in agricultural areas is the primary necessity for any sector-primarily based implementation together with estimating agricultural subsidies. Improved decision remote sensing image currently offer higher useful geographic records to delineate regions; however, their automatic managing is tedious. Its miles therefore critical to increase strategies that permit this activity to be completed right away. In any such process, a novel approach named improving the Enhanced Gustafson-Kessel-Like clustering (EGKL) version explores the use of a pc-mastering device to define agrarian areas. The current method seems for limits as either segment corners or linear traits are adjoining regions of small variation all the time series. Nearby everyday deviations from all images a while are coupled, ensuing in a sequence of extended directional edge filters. Even though, in order beautify the excellent of boundary delineation, this advised paintings is merged with sequential features of small variability across the time collection, which includes the standard deviation (STD), Near-Infra Red (NIR) band, or an index along with the Normalized Difference Vegetation Index (NDVI), or band ratios (particularly for hill us of a), or important component images. A photograph evaluation of the effects obtained with the aid of a methodology relevant to two fields of an excessive-resolution satellite image of the fractured agricultural landscape shows that it is helpful to apply the guide vector machines technique for such a task. Finally, the experimental results reveal that the proposed segmentation method is more efficient than the existing segmentation techniques in factors of each quantitative overall performance metrics and appropriateness for land-use classification.
\end{abstract}

Keywords: Agriculture, Clustering, Function Extraction, Enhanced Gustafson-Kessel-Like Clustering, Image Area Evaluation, Image Segmentation, Photo Series Evaluation, Remote Sensing, Multispectral Edge Detection.

\section{INTRODUCTION}

$T_{r}$ racking agricultural land and estimating plant output is essential for nations whose economy is strongly depending on the agricultural change. This entails not handiest retaining a file of preceding production but also quick-time period

Revised Manuscript Received on December 30, 2019.

* Correspondence Author

Dr. R Priya*, Associate Professor and Head, Department of Computer Science, Sree Narayana Guru College, Coimbatore, India.

Mrs. Darsana, pursuing Ph.D. Computer Science Sree Narayana Guru College, Coimbatore, India.

(C) The Authors. Published by Blue Eyes Intelligence Engineering and Sciences Publication (BEIESP). This is an open access article under the CC BY-NC-ND license (http://creativecommons.org/licenses/by-nc-nd/4.0/) tracking and go back assessment, forecasting agricultural output and informing advertising and buying and selling alternatives. Specifically, up to date plant production facts are received through farmers statements and/or field journeys. This method is not the simplest liable to mistakes and inconsistencies but also time-ingesting and luxurious. Therefore, there may be certainly a requirement for computerized plant ratings. Furthermore, the difficulty has no longer been fixed totally; for accelerated sizes on the level of private regions (ground measurement distance inside the place of one - five $\mathrm{m}$ ), where monitoring with specialized gadgets which includes hyperspectral cameras or polarimetry radar is prohibitively luxurious. Given the increasing deliver for agricultural manufacturing, weather exchange difficulties for agriculture, and monetary pressure, accuracy farming is a developing industry.

The increase and allocation of far off sensing apps are also growing. The accessibility of comprehensive temporal or spatial information is progressed through a remote satellite era and an open-supply approach that gives an attractive chance to gather, compare and use agricultural statistics at the farm scale and beyond. A study performed by Begue et al., [1] confirmed that begin of the season gap between croplands and rangelands differs explicitly in the Mali. These occasions may be used to extract subject limits from satellite imagery. Ming et al. extracted cropland via combining hierarchical rule set-based classification and spatial facts-primarily based suggest-shift segmentation by Ming et al., [2]. Although the strategies referred to above have obtained excellent effects through experiments, most of those at regions that were only blanketed with farmland, which decreased the issue of extraction and lacked universality.

Then again, those techniques used precise or fixed scale parameters, completely have a look at the location, which intended that they have been unable to meet the need of scale dependence and cause the phenomena of mistake extraction or leakage extraction. Consistent with the concept of spatial dependence, the same forms of items frequently have a similar spatial scale and frequently cluster in a nearby area, so images can be divided into a few nearby areas within which the identical gadgets collect. Aiming at the nearby department, researches have offered one-of-a-kind methods in recent years. Georganos et al. [3], [4] used the cutline developing a set of rules and two different regular grid developing strategies to divide the image into local regions.

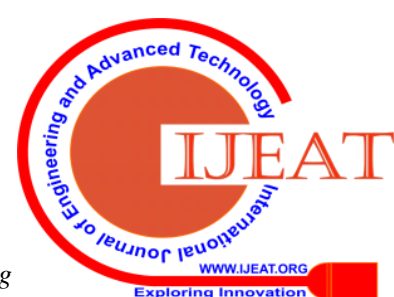


Turker and Kok used [5] cognitive type for computerized far-flung sensing statistics elimination of colorful sub-barriers within contemporary agricultural areas. The approach included discipline boundary facts and satellite images to conduct the sector-based evaluation.

The canny edge detector is used to perceive boundary pixels. Recently in computer vision field techniques, geared toward imitating an expert's demarcation via monitored category strategies have been efficiently implemented to real image segmentation proposed by Nunez-Iglesias et al. [6]. It is also presumed that a similar strategy might be useful for the distinction of agricultural applications. High clarity type of image segmentation, however, the listening is more on image over-segmentation than the optimization of substantial artifacts by Schick and Stiefelhagen [7].

In this appreciate, high clarity computing is not a side in and of itself, but as a substitute as a pre-processing phase in a try to address a sizeable problem, in this example the effective evaluation of an image. High clarity strategies improve photo analysis, e.g. reducing the impact of noise and intra-class spatial variant, keeping maximum corners of images, and enhancing the computing pace of next measures inclusive of segmenting large objects. Super pixel dealing with is accomplished by using an altered variant of the optimization approach referred to as Simple Linear Iterative Clustering (SLIC) [8] that is relied on the well-known k-way technique presented by Achanta et al, to cut up image frames into super pixels. The preliminary SLIC set of rules operates all through the Red Green Blue (RGB) coloration space (defined handiest by way of the pink, inexperienced, and blue spectral bands) and believes parameters: ok, the preferred quantity of high clarity and c, the compactness issue. An extra rate of $c$ emphasizes the significance of geographic closeness arising in greater bendy high clarity.

The SLIC version covered on this task that claims to the carried out by Gonzalo et al [9], which extends the approach to operate with multi-spectral photos. Particularly, techniques relying on segmentation techniques have the previous drawbacks such as: these methods are prone to intra-parcel version that could generate more sections than required and also many of these techniques are extremely conditional at the proper parameter desire (e.g. The resemblance assessed for grouping photograph pixels) requiring preceding know-how of the image or analyses at mistake setting. Also, variation in chart dimensions or paperwork inflicting some settings variables does not permit all applications to be effectively delineated. Lately, in the course of the domain of computer vision, approaches aimed toward imitating the delineation produced by using a specialist through controlled identity techniques have been successfully carried out to real image segmentation. Its miles, therefore, thought that a similar method will be useful for the difference of agricultural barriers.

This process targets to decide whether machine-based strategies can properly train the way to delineate agrarian programs in high-resolution images. In this task, a new method is provided the use of a monitored ranking approach to beautify Enhanced Gustafson-Kessel-Like clustering to delineate agrarian areas. In this respect, a Support Vector Machine (SVM) identity approach is taught the use of a part of a differentiated image underneath research to determine whether neighboring super pixels ought to be combined. Maximum crop facts are computed on a sector foundation, and ranking methods are well established it produces more potent consequences for homogeneous images than according to-pixel rating. A multispectral segmentation technique for computerized delineation of agricultural field limits in remote sensory pixel is provided in this research. This work's primary input is described as follows:

- In this work, the method looks for limits with sequential functions of small variability across the time series, which includes the STD, NIR organization, or indicators which includes NDVI, or band ratios (specifically mountain land), or principal components images.

- Multispectral edge detector uses all available multispectral statistics via integrating the magnitudes and tips of objects obtained from edge detection in a distinct line.

- So created a fresh, streamlined technique for gleaning closed polygons from time-series satellite imagery for pc imagery around areas to beautify Enhanced Gustafson-Kessel-Like clustering (EGKL), orientated model.

\section{PROPOSED METHODOLOGY}

First scanning inside the raster room, observed via transformation to a closing polygon layer of GIS - a depiction of regions as items. The implementation of the approach is described in a time-series satellite imagery sample dataset, show micro-region for corresponding area boundary map that makes use of the cautioned segmentation approach referred to as Enhanced Gustafson-Kessel-Like clustering (EGKL). Then quantitatively and descriptively fit the chart with consequences from any other approach (i.e. Contemporary segmentation tool) completed on the identical photo dataset. The set of rules for delineating the boundaries of agricultural fields is cut up into three components.

a) The sphere boundary detection technique for the evaluation of both rater and Geographic Information Systems (GIS)

b) A hyperspectral edge detection in which the primary limits are observed and properly placed

c) To mix regions from the embedded over-segmentation with the consequences from the brink detection section with EGKL. The suggested research is proven in Fig.1. 


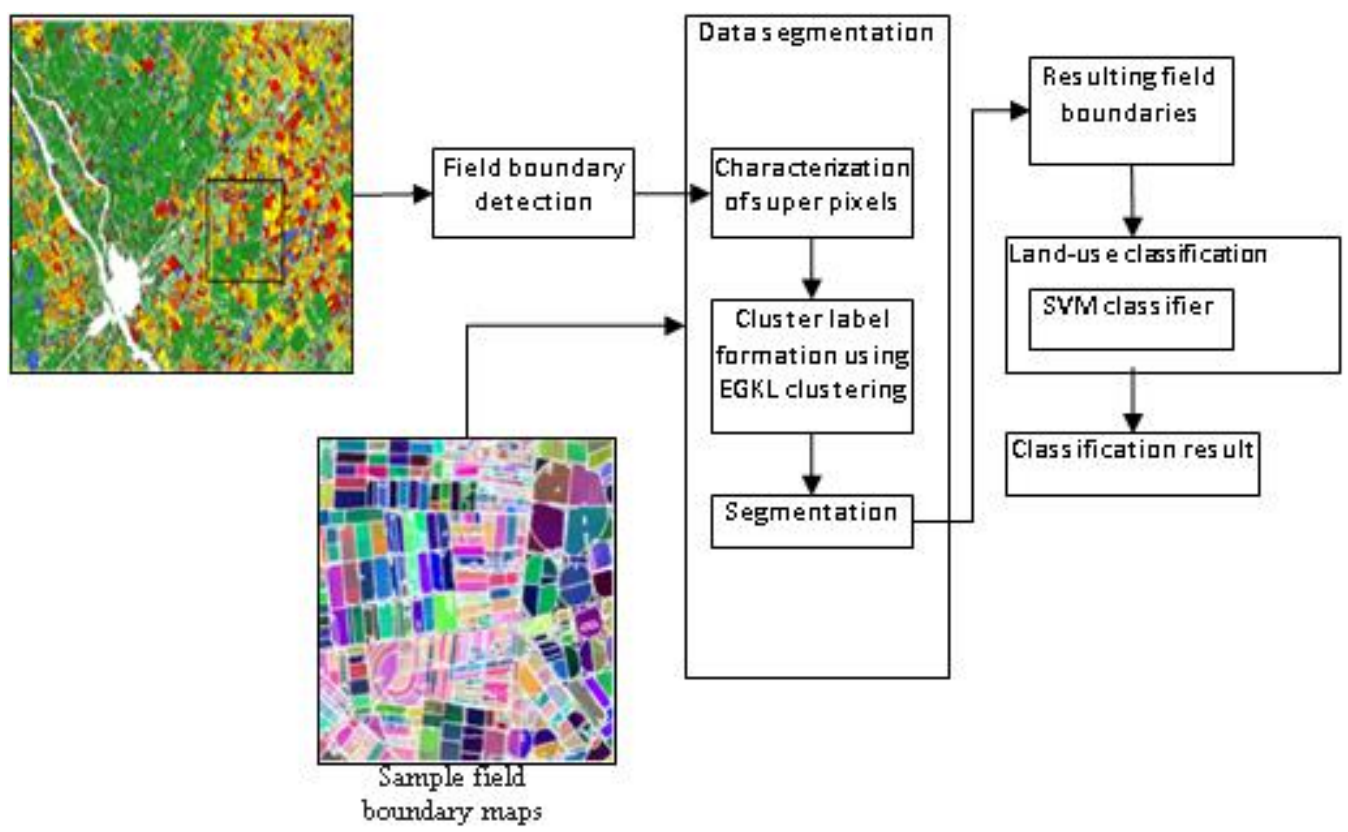

Fig. 1. Proposed methodology of boundary delineation

\section{A. Field Boundary Detection Method for Rater}

The above method works on a satellite photo dataset to increase the probability that any distinct boundary may be great in as a minimum one of the photos. The technique in advance in this thread is proven with records displaying a 6 $\mathrm{km} / 7 \mathrm{~km}$ portion of the entire facts series of experiments outlined. In the interim, its miles enough to note that the pink, Near-Infra Red (NIR) and Short Wave Infra-Red (SWIR) bands from seven dates of spot satellite imagery have been used to generate those figures. Use this collective blend because all of them show useful differences between regions and are relatively uncorrelated. As an instance, it is quite viable to apply only the NIR organization or an indicator including NDVI or band ratios. Underneath are large steps to create the STD photo and to identify long linear capabilities.

1) Earlier than comparing the in keeping with-band STD, a mass can be introduced to restrict their impact to each other. For rare cases where image bands are calibrated to reflectance and used as spectroscopy ratios, they may be already balanced, and have a 1.0 weighting for all bands. However, if the image is designed for a separate set, including radiance, or there is a point of emphasizing one institution over some other, then weighting variables other than 1.0 can be used. In these cases, to perceive the range of weights that might be suitable for an image type (i.e., sensor aggregate, calibration method, and agencies used) in preference to actual photos. To understand one of the groups as a guide (weighting of 1.0) and calculate the ratio of the average dynamic range (over a huge number of images) of this band for a piece other one. If every image includes ${ }^{n}$ bands $b_{k}$, where $\mathrm{k}=1, \mathrm{n}$, just as the weights are $w_{k}$, each array is weighted like $w_{k} \cdot b_{k}$.

2) At every level (and each weighted band) the STD has calculated the usage of photographs in a neighborhood vicinity round that level for each photograph. Here uses a spherical show with a diameter of 5 neurons to discover this neighborhood area (i.e. 21 vertices). Invalid images (e.g. Outside the trajectory of the time-series satellite imagery, contaminated cloud or other areas) do now not add to the STD. This degree will bring about a pile of STD images consistent with the cluster.

3) At each stage, STDs in all photos and agencies have merged the use of an unmarried-layer photograph ratio outlining spatial variability from all photographs and companies. Another time, at this level, the simplest legal photograph STDs are mixed.

4) Linear traits are located within the combined STD image through convolution to 16 regression channel traces, where in each couple contains a left and a correct quarter, $f_{i, \text { left }}$ and $f_{i, \text { right }}$ for $i=1_{, \ldots} \ldots 16$ instructions. Consecutive carriers are $11.25^{\circ}$ apart, so they occupy $180^{\circ}$ together.

5) Using a logical (AND) combo of digital node couples, neighborhood height rows are determined inside the blended STD photograph. The answers of the operators are as in (1),

$L_{i, \text { left }}=f_{i, \text { left }} \otimes S ; L_{i, \text { Right }}=f_{i, \text { right }} \otimes S$

A boundary response meets the following criteria. At each pixel of $B_{i}(x, y)$ as in (2)

$$
B_{i}(x, y)=\left\{\begin{array}{lc}
1 & L(x, y)>0,(L(x, y)+R(x, y))>T \\
0 & \text { otherwise }
\end{array}\right.
$$

Where (x, y) $\mathcal{L} \rightarrow L_{\mathrm{i}, \text { left }}, \mathcal{R} \rightarrow L_{\mathrm{i}, \text { right }}$ is a place of transitional pixels. In the client responses, only an extremely tiny threshold $\mathrm{T}$ is used to show very low-level noise. It is put at a point that seeks to maintain any vital boundary reaction.

6) A related pixel detail evaluation is conducted in every one of the 16 additives the usage of an eight-community to label related line portions.

7) Preferably, components of less than $300 \mathrm{~m}$ lengths are eliminated. The use of an honest number of pixels attached to the piece. To eliminate (sieve) elements of much less than 30 snap shots for $10 \mathrm{~m}$ spot snap shots. The $300 \mathrm{~m}$ boundary becomes selected according to New Zealand's traditional area sizes and layouts.

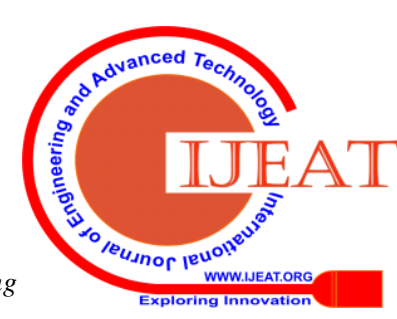


8) All 16 corners are merged into a single layer (binary) coating the usage of a logical OR. As soon as all corners are paired, the row job is extraordinarily linked. Even though our raster evaluation set of rules is ordinarily an edge detection technique, it uses a feature that is more often associated with nearby techniques: that of coloration uniformity, through stretch prolonged linear characteristics. This allows the method to attain the result of an extremely linked course activity. For agricultural regions mentioned that it's far mainly the boundary length of a designated route that suggests that an attitude reaction is vital as opposed to its intensity. The multi-spectral aspect detection is therefore described underneath. Operator associates do now not react to STD gradients (i.e. favorable reaction on the only hand and awful reaction on the alternative) because to calculate each hand individually and assume that each is excellent. This detects the most important rows and therefore continues the client reaction smaller than the whole duration of the STD response. There are also instances when there is extra variation in one portion of a site.

\section{B. Field Boundary Detection Method for GIS}

Raster row studies inside the above measures particularly gives complete boundaries among areas however a few differences stay. The following raster edge map (coupling from all aspects) is transformed into vector layout (GIS) and the last polygon topology is built. Need to bridge these cracks, if necessary, and discard other spurious "dangles" (rows that don't speak on both edge s) till create a GIS sheet to lock polygon topology identifying regions. This next step is, consequently, to function a series of activities (inclusive of several heuristics) using the closed source GRASS GIS [10] to scrub the raster results and transform it into the closing polygon.

1. The usage of an ordinary grass characteristic (skinny), the raster rows are thin to a single image.

2. Urban regions, highways, river beds, ponds, woodlands, hilly and different non-agricultural regions are disguised in raster areas using an individually equipped "agrarian masks." for this mask, here use globally on hand GIS sheets figuring out conservation property, highway networks and huge land cover, observed through guide inspection around small regions such as river banks. Yan and Roy [11] also use cadastral authority's records to separate agricultural property, while on this job fields with continuously improved NDVI, PVI, and SAVI use SVM ranking are detected and the findings are shown in Fig.2.

NDVI: As mentioned before, the Normalized Difference Vegetation Index (NDVI) is the most widely used as VI, which can be expressed as in (3)

$\mathrm{NDVI}=\frac{\mathrm{P}_{\mathrm{NIR}}-P_{R}}{P_{N I R}}+P_{R}$ (3)

PVI: Perpendicular Vegetation Index (PVI) also can be quantitatively expressed as in (4)

$P V I=\left(r P_{N I R}-b\right) \cos \theta-r P_{R} * \sin \theta(4)$

where $\mathrm{wP}_{\text {NIR }}$ and $\mathrm{xP}_{R}$ are the radiation simulated luminance nearby STD. By using the use of prolonged, emphasize and

levels of the NIR and R respectively; ${ }^{b}$ is the intercept of the ground baseline and the vertical surface of the NIR reflectivity, and ${ }^{\theta}$ is the angle between the lateral axis of the $\mathrm{R}$ reflectivity and the ground surface $\mathrm{b}$. In this way, PVI efficiently monitors the impacts of soil context PVI additionally has much less sensitivity to environmental influences and is in general used for the reversal of floor flora parameters (grass output, chlorophyll content), PVI is prone to soil brightness and reflectivity, especially inside the event of small plant life density and calls for to be tailored for this impact.

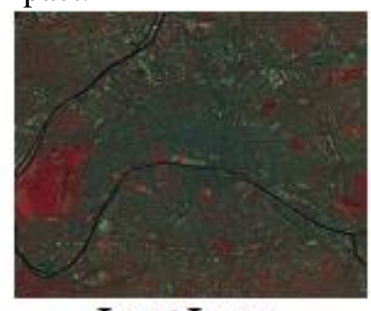

Inp ut Image

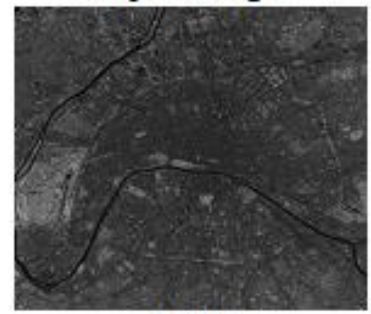

NDVI

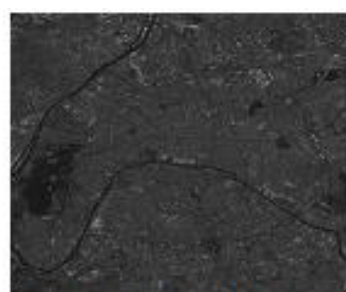

Visible Red Band

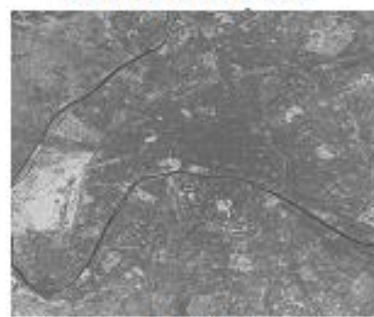

SAVI
Fig. 2.The resultant Bands from the input image

SAVI: Soil-Adjusted Vegetation Index (SAVI) can be expressed as in (5):

$S A V I=\frac{\left(P_{N I R}-P_{R}\right)(1+L)}{P_{N I R}+P_{R}+L}$

The above model of a soil plant life scheme becomes created to decorate NDVI's sensitivity to soil environments, where $L$ is the soil conditioning coefficient, which increases NDVI's sensitivity to soil context. The ${ }^{L}$ range is from 0 to at least one.

1)In sensible apps, ${ }^{L}$ rankings are decided in keeping with instances.

2)While the diploma of flowers exposure is big, ${ }^{L}$ is close to one, demonstrating that the soil context does not affect plant life information extraction.

3)Thin rows are converted right into a vector coating of GIS.

4)Strains are washed to avoid staircases the usage of the GRASS "worms" row generalization technique, with popular parameters.

5)The limits of the agrarian show matrix are carried out. The word that this in valves highways (30 m buffered).

6)The line work is then washed (GRASS v. clean) to avoid duplicates and brief dangles (much less than 1.5 pixels) to decrease the managing burden of step 7 .

7)In their contemporary route, large dangles are increased up to 50 instances their initial duration or $200 \mathrm{~m}$ to permit touch with the primary met row or different expanded cling. 
Here found that these heuristics assure suitable help from the present-day row to disperse throughout most fields that ought to be bridged without integrating spurious boundaries by using increasing the noise-generated brief line section.

8)All closing dangles are segregated, and topology of polygons is built.

9)Polygons out of doors the agricultural location, or less than 1000 square meter are finally flagged as void. When using the area polygons for SVM land-use category to acquire spatial facts in line with the area. This approach excludes spectrally combined pixels from the domain edges.

Edge Detection: The spectral part sensor used for this embedded boundary delineation approach is a gradient-based totally method in which boundary value and orientation are calculated as depicted by Rydberg and Borgefors [12]. Primarily based on the Nevatia-Babu linear edge detector [13], this side detector determines the boundary significance and orientation of a photo utilizing convolution with $5 \times 5$ edge filters, each depicting an angel among 0 and 150 . Vector-wise, the consequences of all six filters are added using measured magnitudes and set filter instructions. Due to the fact, the filter responses are depicted in discrete 30 intervals, a right route is achieved and perhaps the simplest the max-vector is considered, as is the case with the Nevatia-Babu edge detector because the proper course of each the gradient might be between filtration angles. Before including, the vectors are weighted to avoid noise and enhance the precision of the actual orientation of the vector are displayed as follows. The method proposed by Vapnik for adding weighted vectors [14] uses the responses from single masks $\bar{v}_{i}$, which are added as follows to a vector sum $\bar{v}_{\text {tot }}$ as in (6):

$$
\left.\bar{v}_{\text {tot }}=\sum_{i=1}^{n} \omega_{i} \bar{v}_{i}, \omega_{i}=\left(\cos \theta_{i}\right)^{\alpha}, \theta_{i}=\arg \left(\max \left(\bar{v}_{1-n i}\right)\right)-\arg \left(\bar{v}_{i}\right)\right)
$$

Where, ${ }^{w_{\mathrm{i}}}$ is the weight for each particle ${\overline{v_{i}}}_{\text {and }} \mathrm{n}$ is the total number of variables to be introduced. The weights are calculated by using adding the cosine ${ }^{\theta}$ of the range that is the difference between the attitude of the studied vector and the largest vector. The outcome is lifted to which determines the degree of weight. Right here, in the preliminary phase of the set of rules, $\alpha$ is put to 1 to reduce the weighting impact. Adding vectors in instructions between $0^{\circ}$ and $180^{\circ}$ can be done in various respects, resulting in entirely distinct vectors. Using the easy vector sum gradient will attenuate corners for almost inverse gradient parts. That is not acceptable. What is desired is to attenuate $90^{\circ}$ varying reactions. This is the situation if the vector sum is calculated using the double angle, i.e. the value is provided by increasing the filter direction angle. By placing the vector components in this manner, the amount of the vector is both computationally inexpensive and precise. In $\bar{v}_{\text {tot }}$ to this, due to the double angle operation, to be split by two to depict the right angle. When a single edge image is obtained for each spectral band, this boundary data is introduced similarly, but with vectors from output image groups since the orientation of the same object in separate wavelength ranges can be in reverse ways owing to distinct changes from dim to light fields in distinct wavelengths, all boundary angles are transformed to the interval $0^{\circ}$ to $180^{\circ}$.
Right here, to enhance the weighting impact, $\alpha$ is placed to 3 , given that commercial regions hardly ever have tiny sharp attitude corners. For the duration of this manner, lines with reduced importance which are visible in all spectral bands are diagnosed at the same second as noise is attenuated considering noise in all images does not have the same separate route. Such blessings vanish if, as a substitute, the height boundary resistance is utilized in one of the corporations. For the inclusion of the boundary vector from the awesome spectral bands, the wonderful organizations may correctly incorporate statistics from distinct corners. However, smooth surfaces with the same orientation in all businesses ought to have seemed like as an alternative effective boundary. It may be achieved if all part information is taken under consideration and now not simply the maximum. As an instance, in the occasion of a wedge or the passing of a powerful object using a smaller one, it's far essential that the item response is bolstered as it is not an element, or that the stronger element is even extra crushed.

The subsequent boundary photo is then stretched with significance and orientation records, in conjunction with hysteresis thresholding, so that surfaces with a discounted value can be maintained without introducing a vast amount of noise. Small holes within the boundary sections are then crammed in. This algorithm for aspect detection does not result in closed contours. In truth, inside the thinning phase, wherein two perpendicular lines cross, the overlapping pixels are separated. Specifically, gradient-primarily based processing produces surfaces with tender edges and junctions if the filter out extent is larger than three. The thinning technique is supposed to extract these components because the motive of this research is to discover discipline limitations which can be typically recognized to have strong angles. As a substitute, those discontinuities and larger areas wherein the technique disregarded to become aware of corners must be shut by post-processing or the usage of extra procedures, along with a form of optimization. Throughout this activity, segmentation is done by way of improving the Enhanced Gustafson-Kessel-Like clustering (EGKL) technique to derive closed polygons time-series satellite imagery.

\section{Proposed Segmentation Using Enhanced Gustafson-Kessel-Like Clustering (EGKL)}

Segmentation is defined as a method of image separation into homogeneous communities so that every location is discrete, however, neither of the neighboring fields is uniform. The extent of segmentation depends on the favored subject, i.e. As soon as the objects are separated, segmentation needs to be stopped. In this bankruptcy, a sparkling clustering set of rules referred to as the improving GK like (EGKL) protocol is usually recommended that expands on the blessings of the GK algorithm as a treasured clustering approach that may recognize nodes with a shape and alignment.

To cope with the idea of extending the initial GK set of rules is to solve the problem of segmentation in satellite imagery of the rural area boundary delineation. The technique starts with an over-segmentation of the photo obtained through a set of rules of super pixels.

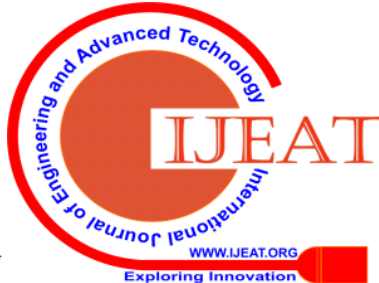


Then perhaps a dataset is advanced from the produced super pixel depiction through acquiring the segment characteristics of every couple of super pixels.

Instances inside the dataset are marked in two businesses based totally on whether or no longer have they corresponded to the equal item (boundary); this fact is furnished with the aid of these days created reference boundary map. The recommended technique makes use of super pixels as minimal dealing with structures, whereas an EGKL approach of super pixels is used to obtain a final segmentation wherein the boundaries (gadgets of the problem) are differentiated. Super pixels are a form of photograph segmentation; however, the concentrate is greater on a regulated over-segmentation. The photograph is, for this reason, break up into many homogeneous regions with a described quantity of pixels. Super pixels can be grouped to gain bigger regions. In this regard, the SVM type approach is taught making use of a part of a segmented scene understudy to decide whether two adjoining super pixels must be blended.

\section{Enhanced Gustafson-Kessel-Like Clustering (EGKL):}

First, it handles only a difficult and speedy amount of facts (extracting characteristics from every couple of remarkable pixels). Second, the set of policies implies the quantity of nodes is recognized earlier. Finally, via the manner of an iterative development device, it determines the nodes. Consequently, in the workout new incoming facts cannot be dealt with by using the initial set of rules system because of the incoming facts movement adjustments over second. The idea of an opportunity recursive clustering set of guidelines just like the concept of the GK set of policies in the revel in of the usage of clusters with a favored information shape is offered in the following. At the beginning, will count on that the GK became implemented to define a unique collection of c nodes of the earlier collected facts. Every one of these nodes is defined through the $\mathcal{C}_{i}$ core and the $\mathbb{F}_{i}$ fuzzy covariance matrix. This speculation is conceivable thinking that some unique statistics about the statistics being treated are obtainable in almost all converting apps. Here its miles assumed that the boundary of every unit is characterized by a manner of the radius of the group calculated from the association ranges $u_{\mathrm{ik}}$ as in (7);

$$
\mu_{\mathrm{ik}}=\frac{1}{\sum_{\mathrm{i}, \mathrm{j}=1}^{\mathrm{c}}\left(\mathrm{d}_{\mathrm{ik}} / d_{j k}\right)^{2 /(m-1)}}, k=1, . ., N
$$

The radius $\mathbb{x}_{i}$ of the ${ }^{i}$ th cluster is equal to the maximal distance ${ }^{d}$ for the ${ }^{i}$ th cluster $d_{i k}$ and $d_{j k}$ between the cluster center $\mathcal{C}_{\mathrm{i}}$ and the points belonging to this cluster with membership degree $u$ larger than or equal to a given threshold membership degree ${ }^{u_{h}}$ as in (8)

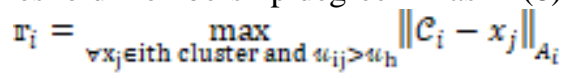

$$
\text { In above equation }\left\|C_{i}-x_{j}\right\|_{A_{i}} \text { is the GK distance between }
$$
$C_{i}$ and $x_{j}$ that norm based on Manhattan distance determined

$$
\left.d_{i k}^{2}\left(x_{k}, c_{i}\right)=\left\|x_{k}-c_{i}\right\|_{A_{i}}^{2} \min _{\substack{1 \leq k \leq 1 \\ 1 \leq i \leq k, 1 \leq k \leq N}}\left(0 x_{k}-c_{i}\|+\| x_{k}-c_{i} \|\right) A_{i}\right)^{I}
$$

The data point $x_{k}$ is assigned to the cluster $p$ if the distance ${ }^{d_{p k}}$ is less than or equal to the radius ${ }^{\mathbb{P}_{p}}$, that is if the condition ${ }^{d}{ }_{p k} \leq \mathbb{x}_{p}$ is satisfied. If this is the case, hence apply the Kohonen rule as proposed by Malone et al [15] to update the $p$ th cluster parameters, cluster center and covariance matrix as in (11)

$$
c_{\text {pinew }}=(1-\alpha) c_{\text {piold }}+\alpha x_{k}
$$$$
F_{p \text { new }}=(1-\alpha) F_{p_{\text {old }}}+\alpha\left(x_{k}-c_{\text {pold }}\right)^{T}\left(x_{k}-V_{p_{\text {old }}}\right)
$$

Where $\alpha=[(0.05 \& 0.03)]$ is a learning velocity; where $\mathcal{C}_{\text {pnew }}$ and $\mathcal{C}_{\text {p.old }}$ are the fresh and old key values; and where $F_{p \text {, new }}$ and $F_{\text {p old }}$ are the fresh and old covariance graph values. If $d_{p k} \leq \mathbb{r}_{p}$ condition runs out, a new potential cluster will be produced, and the number of clusters will increase. Then the incoming data ${ }^{x_{k}}$ is acknowledged as the

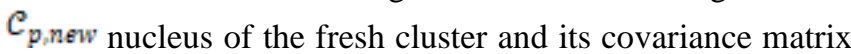
$F_{\text {new }}$ is initialized with the covariance equation of the closes cluster: $F_{\text {new }}=F_{p \text { old }}$. To evaluate the legitimacy of the estimated peaks, a variable $P_{\mathrm{i},} i=1_{x, \ldots} \mathbb{C}$ is introduced to evaluate the quantity of cluster-related items. Its lower certain is anticipated from the minimum quantity of records rows required to understand the parameters of the covariance matrix. It is decided through the dimensionality $\mathrm{n}$ of the statistics vector as $P \min =\mathrm{n}(\mathrm{n}+1) / 2$. A big $P_{\mathrm{i}}$ rating, not most effective guarantees the legitimacy of the covariance matrixes however additionally improves the robustness of the outlier set of rules. It truly is why here are proposing a bigger threshold $P_{\text {toll }}, P_{\text {toll }}>P_{\min }$ matching to the minimum quantity of products located within each group's $\tilde{r}_{\mathbb{I}}^{\pi}$ restrict.

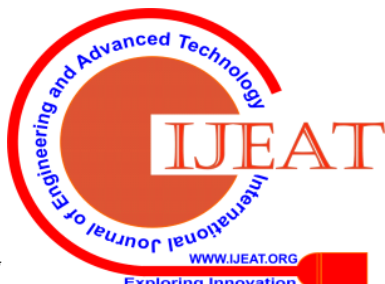


The limit amount is decided with the aid of context due to the specificity of the set of information being taken into consideration. In the earlier step-by way of-step review, the streamlined EGKL summarizing the above ideas is given in Table I.

Table- I: Step by step procedure of EGKL

Initialization: Calculate the initial number of clusters $c$ and the corresponding matrixes $\mathcal{C}, \mathcal{C}$ and $F$; $i=1_{\ldots \ldots} \mathbb{C}$ by the EGKL algorithm. Choose in advance: $\varkappa_{h}, \alpha, P_{\text {toll }}$.

1. Repeat for every new data point ${ }^{x_{k}}$.

2. Calculate $d_{\text {ik }}$ using equation. (9)

3. Determine the closest cluster ${ }^{p:}$ using equation. (10)

4. Calculate the radius $\mathbb{r}_{p}$ of the closest cluster

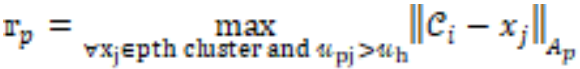

5. If ${ }^{d}{ }_{p k} \leq \mathbb{r}_{p}$ then update the center ${ }^{C_{p}}$ and covariance matrix $F_{p}$ by using equation. (11)

6. Recalculate the partition matrix $u$

7. Else create new cluster $C_{a+1}=x_{g}, F_{a+1}=F_{p}$

8. Calculate the number $P_{\mathrm{a}+1}$

9. If $P_{\mathrm{s}+1} \geq P_{\text {tol }}$

10. Accept the new cluster, $\mathbf{c} \mathbf{c}+1$

11. Else decline the new cluster and keep the old one

12. End

End

\section{Support Vector Machines (SVMs)}

The SVMs are focused on the idea of minimizing environmental hazards by Vapnik [14]. In its fundamental form, an SVM discovers a separate hyper-aircraft between two classes that maximizes the distance among them as proposed by Tso and Mather [16]. The education factors that outline the hyper-plane are known as aid vectors, and the classifier is defined. The coaching specimens are implicitly connected to a higher-dimensional room with a vector function to expand the belief of non-linear choice limits in Vapnik [17]. Hence used the radial base feature (RBF) matrix. Parameters, the kernel period characteristic, and the regularization resistance modify the SVM conduct. To desire the only-against-one technique to restoration multitask trouble with the binary SVM set of rules as it has been recorded to deliver the greater output and decrease training time as depicted by Tso and Mather [16]. SVMs have also been shown to operate well for plant ranking, (Hsu and Lin; Pal and Mather; Pal) e.g., [18],[19],[20]. SVM classification agriculture boundary region detection became performed by using making use of a non-linear classifier on every pixel primarily based on its water indices. In these paintings, the best two training, which might be Agri and non-Agri, have been considered. The SVM became trained in the usage of the pixel and the regarded classes of the training facts. Input given to the SVM was varied through various the wide variety of features selected within the education dataset and by the RBF kernel function utilized by SVM. After training, an independent checking out dataset was classified. To assess the accuracy of the classifier, the space from the testing samples to the isolating aircraft changed into evaluated. The space of misclassified check samples changed compared with the margin rate to decide whether they lied in the risk sector.

\section{EXPERIMENTAL RESULTS AND DISCUSSION}

The whole region of our main test vicinity is the mountains of mid-Canterbury, South Island, New Zealand, a location of pastoral dairy farming (normally irrigated), farm animals and cattle farming (commonly dryland) and agricultural property farming. Land use is dynamic, with widespread farm conversion over the past 10 to 20 years, normally to dairy farms. Now and again this conversion entails wholesale destruction of gift limitations and hedges, set up of large pivot irrigators, and new belongings layouts. It is used it on photograph datasets regular of those used for land-use scheduling to illustrate the segmentation method. Boundary monitoring approach can be carried out to any optical photo. But, to selected pick most effective the larger spatial-resolution SPOT or sentinel-2 pixel (generally seven to 9 images within the 21 month duration) for ground boundary inspection from the images normally used for the land-use assessment level. The method of boundary surveillance may be applied for any optical photo. The red, NIR, NDVI, PVI, SAVI and SWIR bands of each image were used. The vector result had over 91,000 polygons representing fields. This count excludes a slightly larger number of small polygons that would not be considered as a part of a field, such as isolated buildings, trees, vegetation or other small features. For frequently delivered the photograph segmentation the usage of EGKL clustering to a sub-place with the equal image around the beginning fields and the consequences are proven in Fig.3.

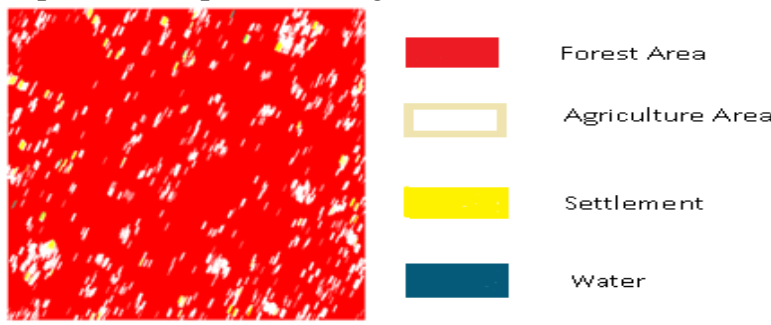

Fig. 3.Segmentation results for the area around the reference dataset

\section{A. PSNR Comparison Results}

The power of the segmentation method became evaluated by the usage of the median of the accuracies for multitemporal satellite images. However, the small segmentation accuracies had been generally caused via the small length of the accumulated photographs in modern segmentation instrument methods which is proposed by North et al [21]. Any such low-decision effect can be determined by using contrasting the accuracies acquired in 3 segmentation results. From Fig.4 the proposed EGKL clustering technique produces a powerful segmentation accuracy result than the prevailing methods, as a result, the PSNR rate is excessive.

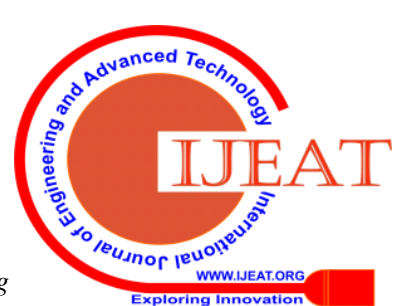


The correct PSNR cost for the proposed EGKL clustering approach is $68.99 \mathrm{db}$ which $18.87 \mathrm{db}$ higher than the present approach.

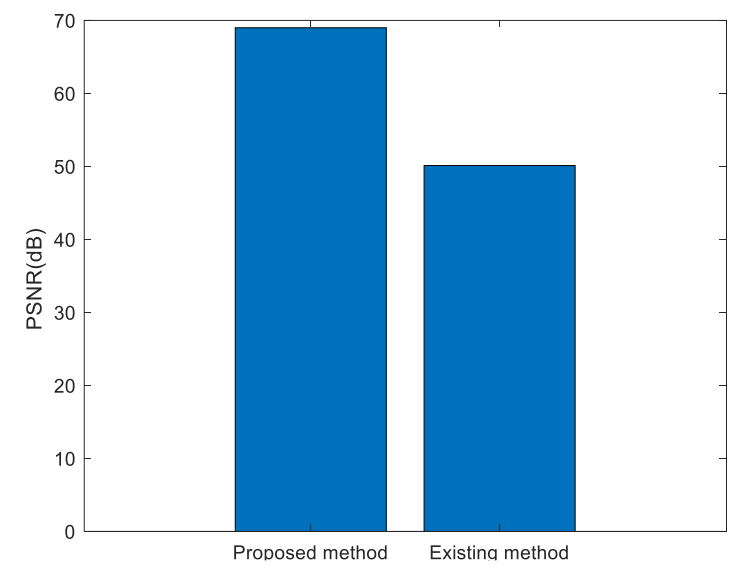

Fig. 4.Segmentation Accuracy Results

\section{B. Processing Time comparison}

From Fig.5, the graph explains that the processing time evaluation for the number of clusters in multitemporal photographs of a satellite. From this graph, it is found out that the proposed segmentation of the usage of EGKL affords lower processing time than the preceding techniques. Therefore, the output explains that the proposed EGKL set of rules is more to the existing algorithms along with segmentation tools in phrases of better boundary delineation results for the rural discipline. The corresponding numerical values are shown in Table II.

Table- II: Name of the Table that justify the values Numerical Values of Processing Time Comparison Result

\begin{tabular}{|l|l|l|l|l|l|}
\hline $\begin{array}{l}\text { Number of } \\
\text { Clusters }\end{array}$ & 2 & 4 & 6 & 8 & 10 \\
\hline $\begin{array}{l}\text { Proposed } \\
\text { EGKL method }\end{array}$ & 8.38 & 9.64 & 12.28 & 11.67 & 14.46 \\
\hline $\begin{array}{l}\text { Existing } \\
\text { segmentation } \\
\text { tool method }\end{array}$ & 11.5 & 12.2 & 14.52 & 15.66 & 16.22 \\
\hline
\end{tabular}

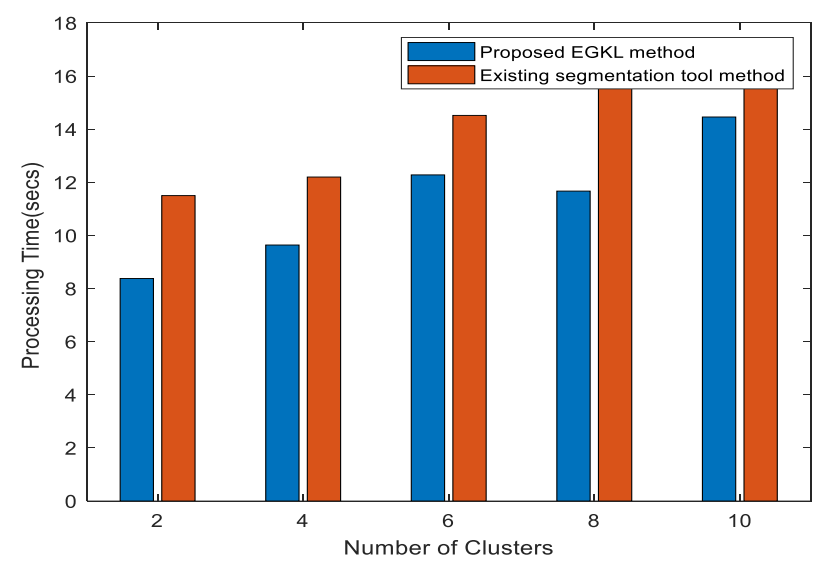

Fig. 5.Result of Overall Processing Time

\section{CONCLUSION AND FUTURE WORK}

The cautioned method utilizes the EGKL clustering algorithm to phase the image to provide super pixels and a supervised type procedure to decide the rural boundary delineation while merging neighboring super pixels. So, got created a segmentation technique custom designed to recognize areas in an agricultural panorama. The method has allowed agricultural assessment on a local scale. This enables the identity of areas as whole objects, which is greater unique than the rating of separate pixels the use of SVM. The location that can be segmented is essentially countless because the photograph is mapped to be processed the usage of the EGKL clustering method. The locational precision of boundary line paintings is about twice the pixel width of the photo used to generate it. Comparison with hand-drawn assessment limits has shown an accelerated diploma of accurate segmentation, implying that the sections hardly ever combine awesome belongings kinds. Both of those findings suggest that the following field obstacles are suitable for entry into a land-use ranking gadget. Evaluation with modern-day segmentation techniques, including the contemporary segmentation instrument and spring, demonstrates straighter, purifier line work, and a result that isn't always disrupted by statistics gaps. The counselled method findings also are illustrated on three New Zealand datasets masking distinct areas, topographies, satellite imagery detectors, and radiometric calibrations. The findings indicated that it's miles possible to educate a teaching device to delineate agricultural packages. The number one trouble of this advised process is the approach of no longer being capable of use partly cloud-covered image and struggling mildly from photographs with an effective tramline mark, resulting in loud images requiring filtering screens. It is going to be adopted as a capacity process to clear up this difficulty.

\section{REFERENCES}

1. . Begue, Agnes, Elodie Vintrou, Alexandre Saad, and Pierre Hiernaux, "Differences between cropland and rangeland MODIS phenology (start-of-season) in Mali," International Journal of Applied Earth Observation and Geoinformation vol. 31, 2014, pp. 167-170.

2. Ming, Dongping, Xian Zhang, Min Wang, and Wen Zhou, "Cropland extraction based on OBIA and adaptive scale pre-estimation," Photogrammetric Engineering \& Remote Sensing 82, vol. 8 , 2016, pp. 635-644.

3. Georganos, Stefanos, Taïs Grippa, Moritz Lennert, Sabine Vanhuysse, and Eléonore Wolff, "SPUSPO: Spatially Partitioned Unsupervised Segmentation Parameter Optimization for efficiently segmenting large heterogeneous areas." In Proceedings of the 2017 Conference on Big Data from Space (BiDS'17), Toulouse, France, 2017, pp. 28-30.

4. Georganos, S.; Grippa, T.; Lennert, M.; Vanhuysse, S.; Johnson, B.; Wolff, E. Scale matters: Spatially partitioned unsupervised segmentation parameter optimization for large and heterogeneous satellite images. Remote Sens. 2018, 10, 1440

5. Turker, Mustafa, and Emre Hamit Kok, "Field-based sub-boundary extraction from remote sensing imagery using perceptual grouping." ISPRS journal of photogrammetry and remote sensing 79, 2013, pp. 106-121.

6. Nunez-Iglesias, Juan, Ryan Kennedy, Toufiq Parag, Jianbo Shi, and Dmitri B. Chklovskii. "Machine learning of hierarchical clustering to segment 2D and 3D images." PloS one 8, vol. 8, 2013, pp. 717-715.

7. Schick, Alexander, and Rainer Stiefelhagen, "Evaluating image segments by applying the description length to sets of superpixels," In 2011 IEEE International Conference on Computer Vision Workshops (ICCV Workshops), IEEE, 2011, pp. 1394-1401.

8. Achanta, Radhakrishna, Appu Shaji, Kevin Smith, Aurelien Lucchi, Pascal Fua, and Sabine Süsstrunk, "SLIC superpixels compared to state-of-the-art superpixel methods," IEEE transactions on pattern analysis and machine intelligence 34, vol. 11, 2012, pp. 2274-2282. 
9. Gonzalo-Martín, Consuelo, Mario Lillo-Saavedra, Ernestina Menasalvas, David Fonseca-Luengo, Angel García-Pedrero, and Roberto Costumero, "Local optimal scale in a hierarchical segmentation method for satellite images," Journal of Intelligent Information Systems 46, vol. 3, 2016: pp. 517-529.

10. GRASS GIS (2017, Aug). [Online]. Available: https://grass.osgeo.org/.

11. Yan, L., and D. P. Roy, "Conterminous United States crop field size quantification from multi-temporal Landsat data," Remote Sensing of Environment vol. 172, 2016, pp. 67-86.

12. A. Rydberg and G. Borgefors, "Extracting multispectral edges in satellite images over argicultural fields," in 10th Int. Conf. Image Analysis and Processing, Venice, Italy, 27-29, Sept. 1999, pp. 786-791.

13. R. Nevatia and K. R. Babu, "Linear feature extraction and description," Computer Graph Image Processing, vol. 13, 1980, pp. 257-269.

14. Vapnik, V. "Statistical Learning Theory"; Springer-John Wiley: New York, NY, USA, 1998.

15. Malone, James, Kenneth McGarry, Stefan Wermter, and Chris Bowerman. "Data mining using rule extraction from Kohonen self-organising maps." Neural Computing \& Applications 15, no. 1 (2006): 9-17.

16. Mather, Paul, and Brandt Tso, "Classification methods for remotely sensed data," CRC press, 2016.

17. Vapnik and Vladimir, "The nature of statistical learning theory," Springer science \& business media, 2013.

18. Hsu, C.-W.; Lin, C.-J. A comparison of methods for multi-class support vector machines. IEEE Trans. Neural Network, vol. 13, 2002, pp. 415-425.

19. Pal, Mahesh, and P. M. Mather, "Support vector machines for classification in remote sensing," International Journal of Remote Sensing 26, vol. 5 2005: pp. 1007-1011.

20. Pal. M, "Support vector machine-based feature selection for land cover classification: a case study with DAIS hyper spectral data." International Journal of Remote Sensing 27, vol. 14, 2006, pp. 2877-2894.

21. North, Heather C., David Pairman, and Stella E. Belliss, "Boundary Delineation of Agricultural Fields in Multitemporal Satellite Imagery," IEEE Journal of Selected Topics in Applied Earth Observations and Remote Sensing 12, vol. 1, 2018, pp. 237-251.

\section{AUTHORS PROFILE}

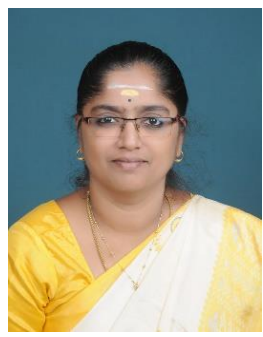

Dr. R Priya, is an Associate Professor and Head in the Department of Computer Science at Sree Narayana Guru College, Coimbatore from December 2000 to till date. She obtained her Ph.D. in Computer Science from Avinashilingam Institute for Home Science and Higher Education for Women in 2015 and also M.Phil. in Computer Science from Manonmaniam Sundaranar University in 2003. Highly focused and has 19+ years of work experience as teaching faculty. 13+ years of expertise in Administration as Head of the Department. Efficient researcher with 15 years of experience and produced 25 M.Phil. Scholars. Her area of interest in Programming Languages include VB, Java and in Web Technologies includes HTML, XML, ASP.NET. She has published 27 International Publications, 4 National Publications, 3 Papers Presentations in Conferences, 19 Seminar/Conferences attended, Acted as Resource Person for 10 Events, 14 Faculty Development Programs attended.

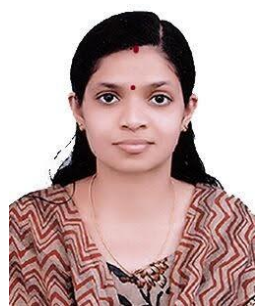

Mrs. Darsana, k Gopidas is pursuing Ph.D. in Computer Science at Sree Narayana Guru College, Coimbatore, India under Bharathiar University. She obtained her M.Phil. in Computer Science from Sree Narayana Guru College, Coimbatore under Bharathiar University. She has 3 years of teaching experience. Her areas of interest include $\mathrm{C}, \mathrm{C}++$ Java, .NET. She has published 2 International Publications. 